\title{
Key Areas in the Endourology, Laparoscopic and Robotic Urologic Surgery Learning: A Resident Spanish Multicentre Survey
}

Hector Garde Garcia*, Elena Ortiz Oshiro, Marco Ciappara, Liz Poma Medrano, Manuel Fuentes Ferrer, Vicente Vera Gonzalez and Jesus Moreno Sierra

Clinico San Carlos Hospital, Madrid, Spain

\begin{abstract}
Objective: To determine the current state of training of urology residents in Spain in laparoscopic surgery and robotic urologic surgery

Methods: At the Department of Urology of the Clinico San Carlos Hospital in Madrid these methods were surveyed and directed to Spanish residents, during the period between 2011 and May 2012. We designed a survey that was disseminated through the website (www.seclaendosurgery.com) of the Spanish Society of Laparoscopic and Robotic Surgery (SECLA) to all intern doctors at any Spanish hospital

Results: Of a total of 384 residents in Urology, 36 responded to the survey $(9.3 \%)$. The data related to endoscopic procedures showed that $25 \%$ of respondents have never participated in the placement of a percutaneous nephrostomy $(n=36)$, or expected to. By contrast, $77 \%$ say that they have done it as surgeons in ureteroscopy $(n=36)$ and $25 \%$ did it in more than ten procedures. The $54{ }^{\prime} 4 \%$ of respondents have participated as surgeons in percutaneous nephrolitectomy $(n=28)$ and $79 \%$ expected to do it. The participation of residents in assisted procedures with the da Vinci robot is low. Laparoscopic procedures are of great interest and participation is reduced on increasing the complexity of the procedure. $41.6 \%$ of respondents think that their training is adequate while $58.3 \%$ think it is not. $88 \%$ think that their training could be improved with courses and seminars and by gaining more responsibility in the operating room and $50 \%$ that their training could be supplemented with external rotations and/or fellowships
\end{abstract}

Conclusion: It is necessary to define what the best ways are to start in laparoscopy surgery and to improve participation in basic laparoscopic procedures to improve the residents' skills and to get the right training in more difficult surgeries. The participation in endoscopic procedures is acceptable.

Keywords: Surgical education; Laparoscopy; Robotics; Endourology

\section{Introduction}

The rapid development of minimally invasive surgery coupled with the complexity of the different techniques is a challenge when training new specialists, especially if you consider that formed urologists have not completed their learning curve yet. Moreover, the increase of urology residency positions, offered by government agencies, even in centres where there is no chance of getting training in these techniques, resulting in a diversification of surgical treatment of different pathologies and heterogeneous ways in the manner of treating. This causes a decrease of the number of minimally invasive surgeries achievable by a resident physician, being undermined by the quality of their training $[1,2]$.

In order to remedy this situation that limits the training activity of resident physicians some activities have been devised. This is the case of simulation, proper animal legal requirements, courses, seminars, rotations and fellowships could lead to fill the gaps we face today [3].

\section{Material and Methods}

The aim of this study is to analyse the current situation of resident trainees in minimally invasive surgery (CMI) in our country.

In the Department of Urology of the Clinico San Carlos Hospital in Madrid, a survey has been designed to all residents of urology of the Spanish hospitals, promoted by the Spanish Society of Laparoscopic and Robotic Surgery (SECLA), through its website (www.seclaendosurgery. es). The survey was divided into four blocks (Figure 1): 1) demographics, 2) general information about the hospital 3) participation in the various endoscopic techniques, laparoscopic and robotic, both present and future expectations of participation, and 4) Aspects of satisfaction with training and areas for improvement.
A descriptive study was conducted with the answers of the respondents.
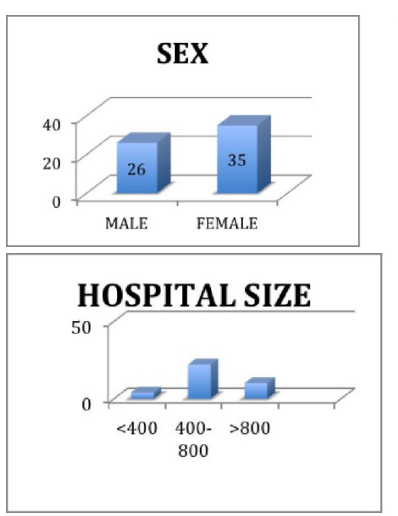

Figure 1: Demographics.

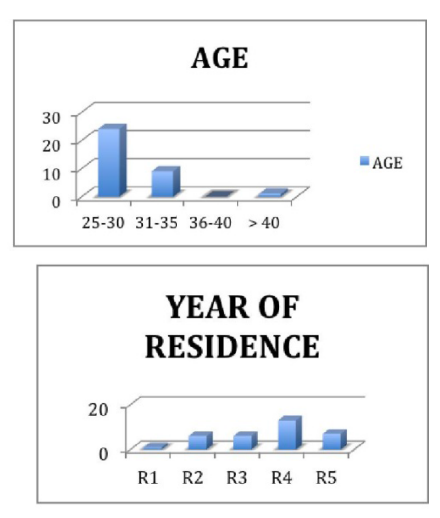

YEAR OF RESIDENCE
*Corresponding author: Hector Garde Garcia, Clinico San Carlos Hospital Madrid, Spain, Tel: 0034 645188870; E-mail: hector.gardegarcia@gmail.com

Received November 11, 2013; Accepted December 22, 2013; Published January 02, 2014

Citation: Garcia HG, Oshiro EO, Ciappara M, Medrano LP, Ferrer MF, et al (2014) Key Areas in the Endourology, Laparoscopic and Robotic Urologic Surgery Learning: A Resident Spanish Multicentre Survey. Surgery Curr Res 4: 160. doi:10.4172/2161-1076.1000160

Copyright: (c) 2014 Garcia HG, et al. This is an open-access article distributed under the terms of the Creative Commons Attribution License, which permits unrestricted use, distribution, and reproduction in any medium, provided the original author and source are credited. 


\section{Results}

The survey (Figure 1) was answered by resident physicians invited to participate voluntarily in the study $(36 / 384,9.4 \%)$.

\section{Demographic data (Figure 2)}

Of the 36 people who answered the survey 26 were men $(72.2 \%)$ and 10 women $(27.7 \%)$ and most of them $(n=35)$ were in the age range between 25 to 35 years. In this study, the most represented group was the resident of the final years of training (fourth and fifth years) with a total of $22(61.1 \%)$ people, followed by the second year $(6 / 36,16.6 \%)$ and third year $(6 / 36,16.6 \%)$.

We analysed the data according to hospital size, ranging from a high percentage of respondents to medium-sized hospitals $(22 / 36$, $61.2 \%)$ and large $(10 / 36,27.8 \%)$ ones.

\section{Formation (Figure 3)}

Of the residents surveyed, we can say that 50\% (18/36) has experimental surgery laboratory and only $7 / 36$ (19.4\%) have the option of performing animal simulation.

25 people answered that at their centre one course per year of laparoscopy is organized at least. Robotics courses are conducted only in teaching hospitals that offer da Vinci robot or training centres.

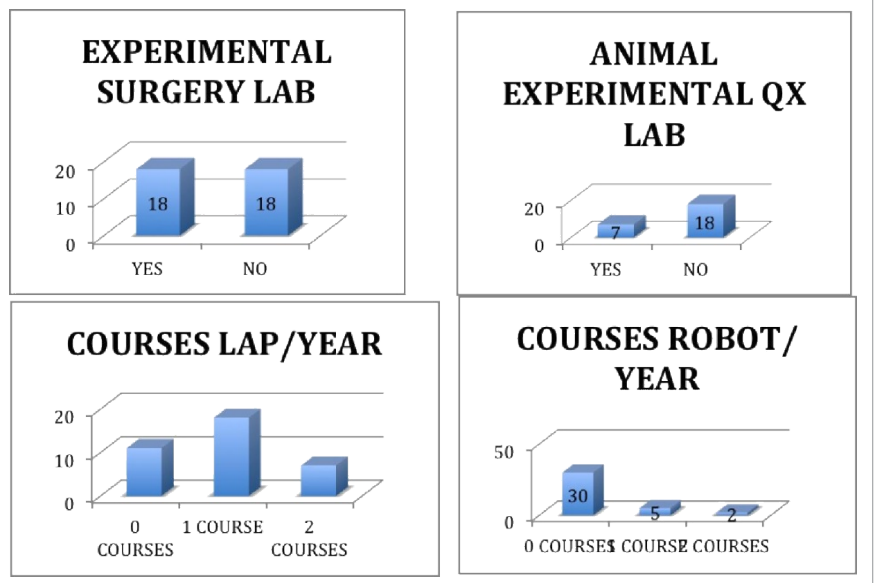

Figure 2: General information about the hospital.

\section{YOU THINK YOUR TRAINING IS ADEQUATE?}

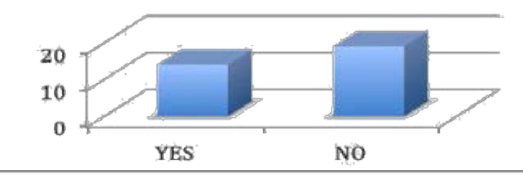

HOW DO YOU THINK COULD BE IMPROVED

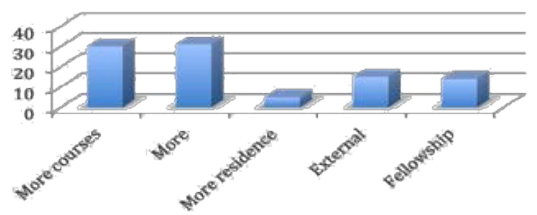

Figure 3: participation in the various endoscopic techniques, laparoscopic and robotic, both present and future expectations of participation.

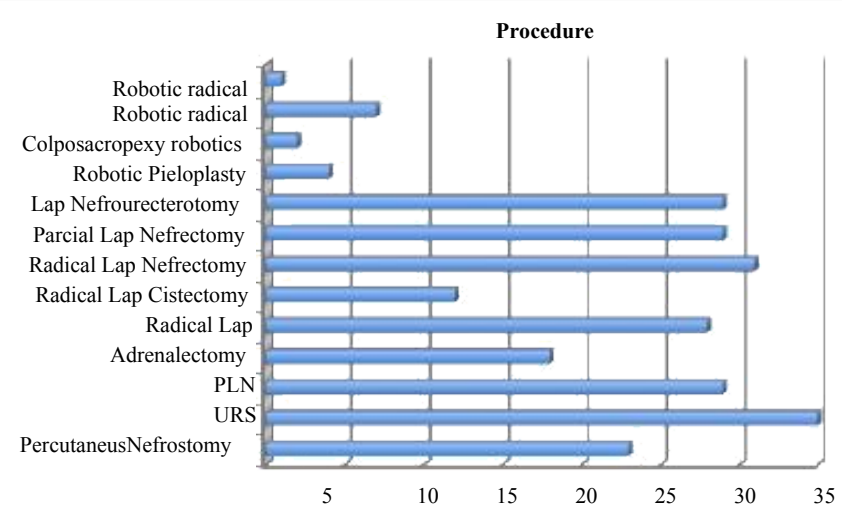

Figure 4: Aspects of satisfaction with training and areas for improvement.

To the question, do you think your training is appropriate? $21 / 36$ people (58.3\%) have answered "no" and 31/36 (86.1\%) of the physicians surveyed think that could be improved in the following ways: 1) by strengthening the organization of courses 2) by providing greater responsibility to the resident in surgery and 3) 50\% (18/36) with external rotations and/fellowships (Figure 4).

\section{Procedure}

Describes the procedures performed by residents described in the polls. We can see that robotic surgery is a technology available in few centres and laparoscopy is used in a variety of pathologies. However, complex procedures exist (cystectomy, etc.) that are not performed at all centres, instead, procedures endoscopy are performed in almost all hospitals.

\section{Endoscopic procedures}

Percutaneous nephrostomy: This technique is frequently performed by interventional radiologists. Of 36 respondents, 9 people $(25 \%)$ or have participated in the making of a nephrostomy, or believe that they will in their training period. More than half have participated only between one and five times in the last year.

Ureteroscopy: Of the 36 respondents, 28 people (77\%) haveparticipated as surgeons, one third of them over 10 times, keeping expectations of participation close to $100 \%$.

Percutaneous nephrolithotomy: Of 27 respondents, 17 (58.4\%) participated as surgeons and $23(79 \%)$ expect to do it. All respondents expected to participate at least by assistantships.

\section{Laparoscopic procedures}

Radical nephrectomy: Of 33 respondents, 28 people (84\%) performed assistantships and 12 people (36\%) did between one and five procedures as surgeons, staying these expectations for the future.

Partial nephrectomy: Of the 28 respondents, 15 people $(54 \%)$ never participated and 12 people (42\%) do not expect to do it in the future.

Nephroureterectomy: Of 31 respondents, 50\% never participatedand $50 \%$ usually do assistantships. 28 people (90\%) expected to perform even 11 assistantships (35\%) or expected to do some over their residence.

Radical prostatectomy: Of 29 respondents, 22 people (75\%) haveassistantships and 7 people (24\%) performed some. Up to 10 people (34\%) think that they will do it in the future. 
Radical cystectomy: It takes only a few centres and only 14 peoplehave answered this section of the survey. 24 people (66\%) or have participated or are hoping to do it. 10 people out of the 14 , performed assistantships and hope to continue doing them.

\section{Robotic procedures}

Only 8 of the respondents have had contact with robotics by low availability performing assistantships, being the most performed procedure in radical prostatectomy. Of the 8 doctors surveyed, 4 were involved between 1 and 5 times as an assistant, one between 6 and 10 and 1 in 10 .

\section{Discussion}

Among the difficulties that we have regarding the very formation of a urology resident, some aspects must be emphasized such as: 1) the rapid development of complex surgical techniques that increase the learning curve, 2) the limitation to gain experience related to the increased supply of places 3 ) the volume of cases is limited in many centres, 4) Need for continuous training of specialists and 5) need to maintain skills acquired simultaneously with learning new skills [4]. The five parameters mentioned that making progress in the acquisition of skills is not easy.

In other professional areas there are very complex simulators that allow professional training in all possible conditions before facing a real situation. In our environment we have the added difficulty that the complexity of human beings is very difficult to simulate considering that sooner or later we will have to face a real patient.

There are different types of simulators for developing and improving our skills, both low fidelity (the models based on a box that develop basic skills) and high fidelity that attempt to represent reality to details cause emotions and generate responses that would be observed in a real situation (as in virtual reality devices or animal practices).

Although much of the learning is based on the repetition of procedures other things are needed to acquire the degree of competence as Urologists.

Focusing on our survey, we were at first struck by the low participation rate $(36 / 384,8.4 \%)$, however we do not believe that this is due to lack of interest in the topic, but rather to the lack of confidence change in opinion polls may cause or lack of time.

Looking at the profile of respondents, most residents were in their fourth and fifth year $(22 / 36,61.1 \%)$ which is consistent with a greater interest in the topic as they are nearly coming to the end of their training period.

As for the procedures performed, the most performed are endoscopic result consistent with longer use of different techniques, however, we have to be careful with the "interference" that we can present toother specialties in the traditionally performed by urologists techniques such as percutaneous nephrostomy. Laparoscopic techniques are spreading rapidly, but when formed, complex ones such as radical prostatectomy still have difficulty getting access. The technique in which there is more participation in the nephrectomy, the number of assistantships is satisfactory ( $84 \%$ ) but the number of people performing some before finishing their training period seems inadequate (36\%). Given the low availability of robotic technology in our country, we think that the best way to contact would be by doing rotations for units that have it.

We know that an urologist comes from the mixture of the acquisition of basic knowledge about manual skills and clinical judgment. The transmission of knowledge is not easy and is a real challenge in medical schools and hospitals. Insisting on this concept, we know that an operation has two basic components: 1) a $25 \%$ skill or skills acquired and 2) a $75 \%$ of successful decision making [5]. This means that the cognitive area is often undervalued in the Urologist surgical education.

The residents are special kinds of students who should know why they learn, what to learn and how to learn it. Therefore, it is a key part of their own training and they must have willingness and be oriented towards problem solving. We look forward to acquiring knowledge and skills to implement them efficiently in clinical practice, this can be valued as strong points in our favour but we must be also aware of our weaknesses and deficits in self-criticism, etc [6].

Looking at the data from the survey, it is difficult with these levels of participation to be able to perform surgeries automatically.

Well, if we have the ingredients: What is a good surgeon?, What can the resident be offered?, How could we improve resident training?

We believe the plan should really be the same in all hospitals in the country and that all residents should have a minimum of working hours in simulators complemented with courses that allow them to acquire the basic skills and enough knowledge to enable them to deal with guarantees for clinical practice.

If we focus on the survey, this is not satisfactory, so we want to draw attention to this aspect.

Anyway formation process must be a homogeneous and standardized process, strongly supported by government agencies, scientific societies and guardians. Above all, in order to improve the shortcomings and weaknesses to end on an assessment and get an accreditation to ensure successful completion of the objectives

\section{Conclusion}

It is necessary to standardize surgical learning to improve the residents' skills in minimal invasive procedures and to get the right training for performing more and more difficult procedures by gaining confidence and giving a safer response to the patient.

\section{References}

1. Mamut AE, Afshar K, Mickelson JJ, Macneily AE (2011) Surgical case volume in Canadian urology residency: a comparison of trends in open and minimally invasive surgical experience. J Endourol 25: 1063-1067.

2. Palter VN, Orzech N, Aggarwal R, Okrainec A, Grantcharov TP (2010) Resident perceptions of advanced laparoscopic skills training. Surg Endosc 24: 2830 2834.

3. Shay BF, Thomas R, Monga M (2002) Urology practice patterns after residency training in laparoscopy. J Endourol 16: 251-256.

4. Preston MA, Blew BD, Breau RH, Beiko D, Oake SJ, et al. (2010) Survey of senior resident training in urologic laparoscopy, robotics and endourology surgery in Canada. Can Urol Assoc J 4: 42-46.

5. Spencer $F$ (1978) Teaching and measuring surgical techniques: the technica evaluation of competence. Bull Am Collsurg 63:9-12.

6. Norman GR (1999) The adult learner: a mythical species. Acad Med 74: 886889 